\title{
Quantification of Strand Accessibility in Biostable DNA Origami with Single-Staple Resolution
}

\author{
Alexandra S. Eklund, ${ }^{\#}$ Alice Comberlato, ${ }^{\#}$ Ian A. Parish, Ralf Jungmann,* and Maartje M. C. Bastings* \\ Cite This: ACS Nano 2021, 15, 17668-17677 \\ Read Online
}

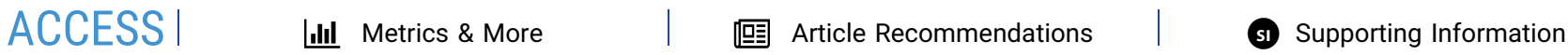

ABSTRACT: DNA-based nanostructures are actively gaining interest as tools for biomedical and therapeutic applications following the recent development of protective coating strategies prolonging structural integrity in physiological conditions. For tailored biological action, these nanostructures are often functionalized with targeting or imaging labels using DNA base pairing. Only if these labels are accessible on the structure's surface will they be able to interact with their intended biological target. However, the accessibility of functional sites for different geometries and environments, specifically after the application of a protective coating, is currently not known. Here, we assay this accessibility on the level of single handle strands with two- and three-dimensional resolution using DNA-PAINT and show that the hybridization kinetics of top and bottom sides on the same nanostructure linked to a surface remain unaltered. We furthermore

DNA-PAINT of K10PEG-coated DNA origami

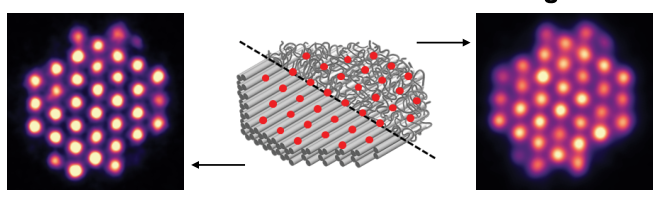

Top and bottom accessibility and 3D measurement

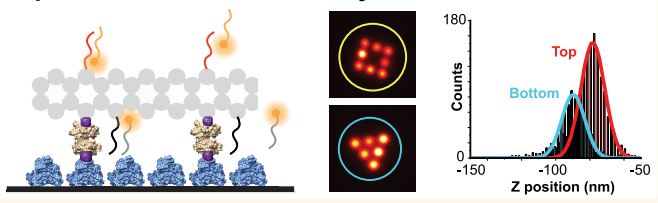
demonstrate that the functionality of the structures remains available after an oligolysine-PEG coating is applied, enabling bioassays where functionality and stability are imperative.

KEYWORDS: DNA origami, DNA-PAINT, super-resolution microscopy, structure stability, handle accessibility, nanotherapeutics, nanopatterns

$\mathrm{T}$ he potential of using synthetic DNA as a biomaterial has gained considerable engineering interest over the past decades and initiated the field of DNA nanotechnology. ${ }^{1}$ Following recent advances in mass production ${ }^{2}$ as well as the development of multiple strategies to stabilize structures in physiological fluids, ${ }^{3}$ the utility of DNA-based nanomaterials for biomedical applications such as drug delivery $^{4}$ and vaccination ${ }^{5}$ is currently being explored. Structures can be functionalized with small synthetic or natural molecules including drugs, targeting ligands, and imaging labels, using a classical "handle/anti-handle" (H/AH) hybridization approach ${ }^{6}$ (Figure 1a). However, quantitative singlemolecule data on whether these functional sites are still available after structural protection using stabilizing coatings are currently missing.

Reports on the yield and accessibility of DNA handle functionalization remain limited to qualitative assays ${ }^{7,8}$ or nonprotected structures. ${ }^{9-11}$ Analytical techniques used to visualize the successful functionalization of handle-decorated DNA origami (including AFM, ${ }^{12} \mathrm{TEM}^{13}{ }^{13}$ DNA-PAINT, ${ }^{10}$ or correlative approaches ${ }^{14}$ ) have furthermore been limited to probing a single active surface of the structures (e.g., the top surface after structure immobilization using binding entities on the bottom surface). However, it is becoming increasingly important to target and visualize both top and bottom surfaces of sheet-like DNA origami for cellular applications, especially when structures are used to interact with molecules on cell membranes. Here, the top (or active) surface could be ligandfunctionalized, while the bottom surface might be used for barcoding, to determine the structure's identity and exact position. Many natural biointerfaces adapt a multivalent interaction of ligand-receptor pairs to convey extracellular information cues for downstream cellular signaling and response. ${ }^{15,16} \mathrm{~A}$ dual-sided nanopatterned disk design would allow interference on both sides of a reciprocal messenger pathway, while simultaneously offering accessibility for barcoded structure visualization by fluorescence microscopy.

Received: June 29, 2021

Accepted: September 30, 2021

Published: October 6, 2021 
a
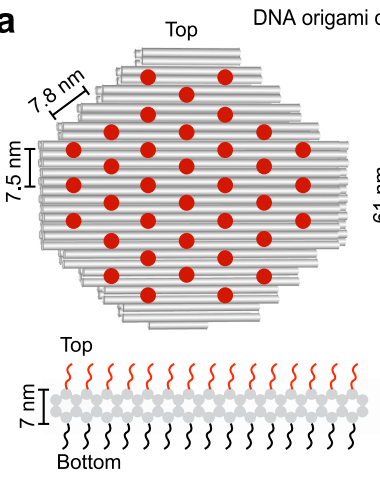

d
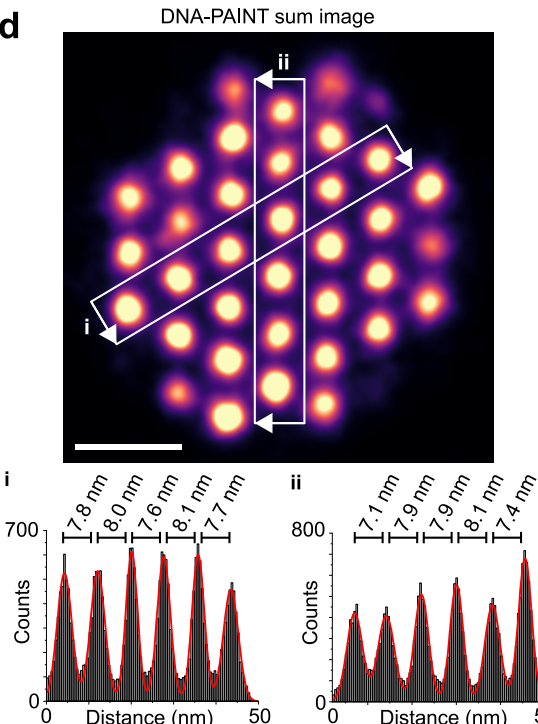

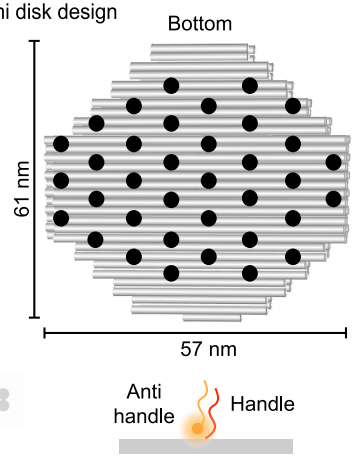

e

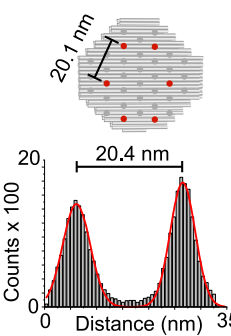

g

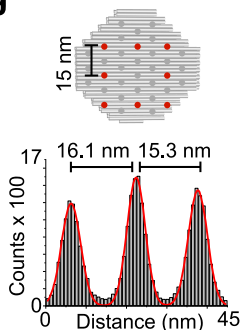

b
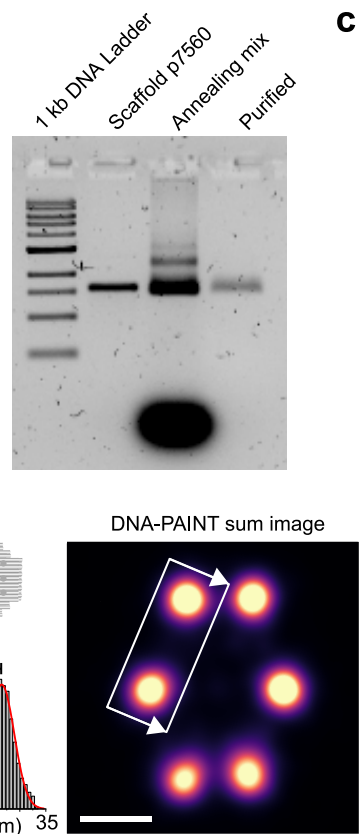

DNA-PAINT sum image

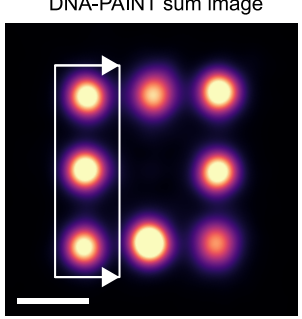

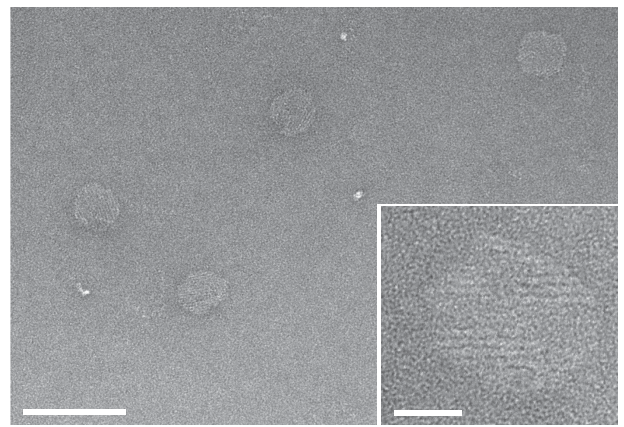

f

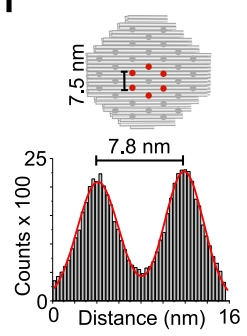

h

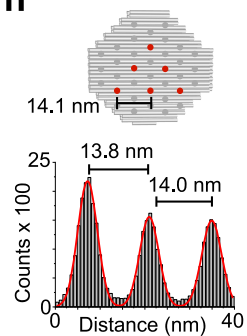

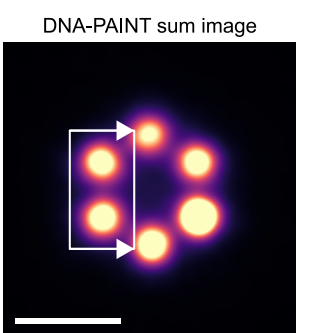

DNA-PAINT sum image

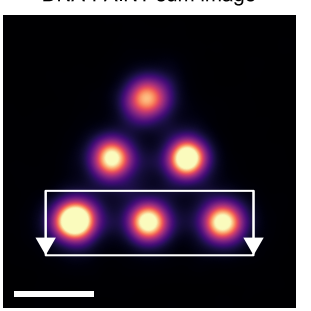

Figure 1. DNA origami disk design and introduction of strand modifications. (a) (Top) Schematics and in silico distances of the DNA disk, top and bottom view with 36 functionalization points per side. DNA-PAINT handles available on the top side (red spots) or bottom side (black spots) of the disk are indicated. Side view (bottom left), showing the six-helix bundle with a thickness of $\sim 7 \mathrm{~nm}$. Functionalized antihandle and handle representation (bottom right). (b) Agarose gel electrophoresis of a DNA disk. (c) TEM images of a purified disk and zoom-in of a selected disk (bottom right). (d) DNA-PAINT sum image of the top of the fully functionalized disk (top) and cross-sectional histogram analysis (bottom) of the highlighted areas, revealing average distances of $7.9 \mathrm{~nm}$ on the diagonal (i) and $7.7 \mathrm{~nm}$ on the vertical line (ii); 150 DONs were used for this sum image. $(e-h)$ Schematic representation of four different pattern designs with DNA-PAINT single-stranded extensions indicated in red (top left). DNA-PAINT sum image of the different patterns: large hexagon (e), small hexagon (f), square $(\mathrm{g})$, and triangle (h) (right); 100-150 DONs were used for the different sum images. Cross-sectional histogram analysis for the highlighted areas and measured distances of $20.4 \mathrm{~nm}(\mathrm{e}), 7.8 \mathrm{~nm}(\mathrm{f}), 15.7 \mathrm{~nm}(\mathrm{~g})$, and $13.9 \mathrm{~nm}(\mathrm{~h})$ matching the simulated distances (bottom left). Scale bars, $100 \mathrm{~nm}$ (c overview), $20 \mathrm{~nm}$ (c zoom-in), and $15 \mathrm{~nm}(\mathrm{~d}-\mathrm{h})$.

Furthermore, none of the characterization techniques have been applied to coated DNA origami nanostructures (DONs), which are essential to ensure structural integrity in cellular applications under physiological conditions. To date, one of the most promising methods to achieve stability of DONs in physiological conditions (e.g., fluids with low divalent cation concentration and high nuclease activity, as found in standard cell culture medium and blood circulation) remains the oligolysine-PEG polymer coating (K10PEG). ${ }^{17}$ Here, an oligolysine polypeptide electrostatically wraps around the DNA helix, effectively neutralizing the negative backbone charge, while a short PEG tail blocks the degradation attack from circulating nucleases. This strategy has shown to stabilize DONs for over $48 \mathrm{~h}$ in harsh conditions without detectable cellular toxicity, making them useful tools for nanomedicine applications. Functionalization with the cell-targeting RGD peptide showed a 2.5 -fold increase in $\mathrm{DON}$-cell interactions, but this analysis presented a mere qualitative view of handle availability. ${ }^{17}$
Here, we use DNA-PAINT super-resolution microscopy ${ }^{18,19}$ to visualize $\mathrm{H} / \mathrm{AH}$ geometrical patterns on a double-faced DNA origami disk and quantitatively assess their accessibility and hybridization kinetics on both sides of the nanostructure at the single-handle level using highest-resolution two- and threedimensional DNA-PAINT microscopy. Our study furthermore explores the compatibility of DON protective coatings with handle availability through precise quantification of the hybridization kinetics of functionalized DNA origami nanostructures using DNA-PAINT.

\section{RESULTS AND DISCUSSION}

Geometrical Patterns on a Double-Faced DNA Origami Disk. Cellular interactions in nature are often marked by the local organization of ligand-receptor binding events in a multivalent fashion. For example, T-cells and dendritic cells exchange information on tolerance or infection through the spatiotemporal immune synapse. ${ }^{20}$ The interaction geometry of two spheres that touch each other is a flat disk on 

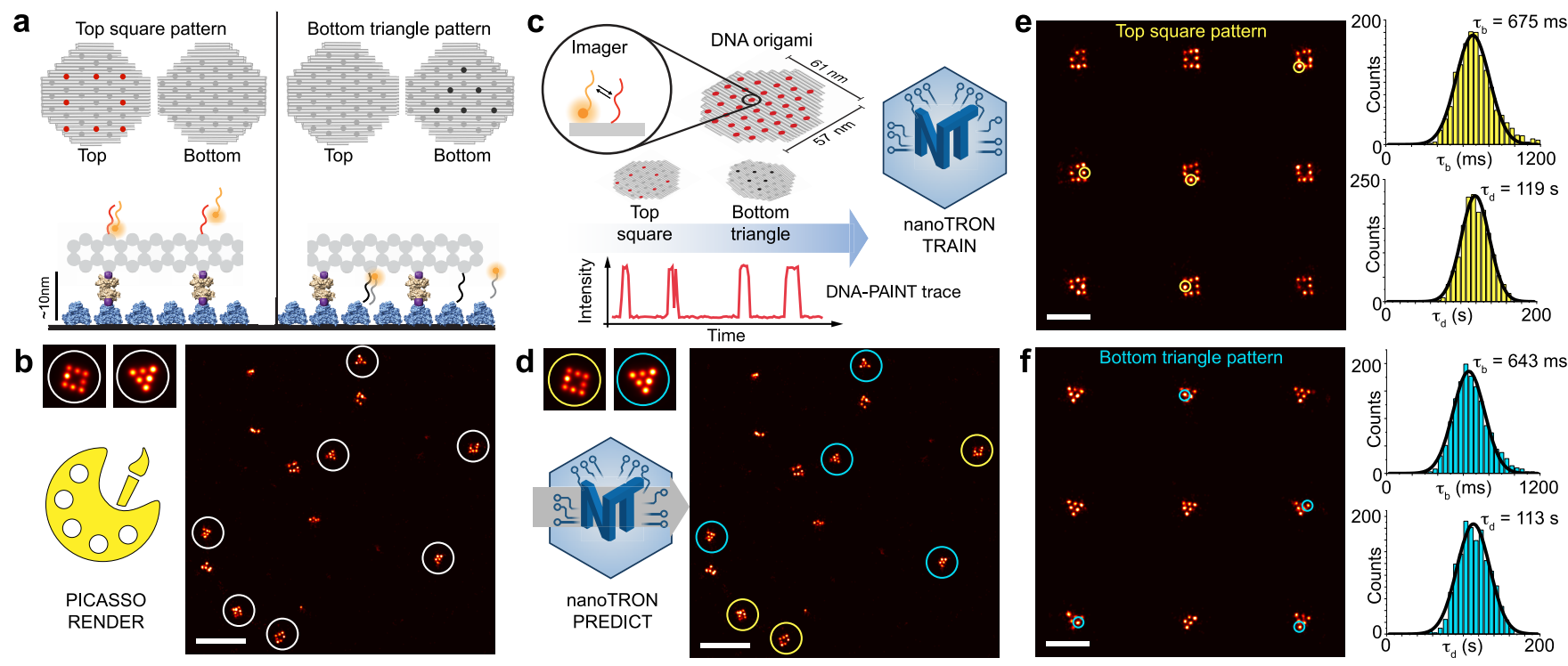

Figure 2. Accessibility and binding kinetics of the top and bottom of the disk. (a) (Top) Schematic representation of the two different origami designs "top square" and "bottom triangle" used for kinetics calculations. DNA-PAINT docking sites for the P3 imager are depicted for the top square (red) or bottom triangle (black). (Bottom) Side-view representation of the disk attached to the slide via biotinneutravidin interactions with the same imager binding either to the top or bottom of the disk. (b) (Top left) Sum image of the squares and triangles used for nanoTRON training. (Right) Field of view where both top square and bottom triangle origami are present. Both patterns were selected (white circles) for further analysis using nanoTRON. (c) Schematic representation of the nanoTRON training process. ${ }^{24}$ (d) Field of view where DONs were classified into square (yellow) or triangle (cyan) patterns using nanoTRON predict. (e, f) Example overview image of structures where single sites were selected for kinetic analysis of the top square pattern (e) or bottom triangle pattern (f). Bright time (top) and dark time (bottom) distribution for imagers binding to the top square pattern (e) or bottom triangle pattern (f) and corresponding Gaussian fits $(n>1000)$. There were no significant differences between top $\left(\tau_{\mathrm{b}}=653 \pm 43 \mathrm{~ms}, \tau_{\mathrm{b}}=130 \pm 27 \mathrm{~s}\right)$ and bottom kinetics $\left(\tau_{\mathrm{b}}=623 \pm 33 \mathrm{~ms}, \tau_{\mathrm{b}}=126 \pm 33 \mathrm{~s}\right)$. The experiment was repeated three times independently with similar results. Scale bars: 200 $\mathrm{nm}(\mathrm{b}, \mathrm{d}), 100 \mathrm{~nm}$ (e, f). NanoTRON logos were modified from ref 24.

the nanoscale, which we used as inspiration for this study. Using the DNA origami approach, ${ }^{21}$ we designed a $60-\mathrm{nm}$ diameter disk (Figure 1, Figure S1 and Figure S2) featuring a six-helix bundle cross-section, which yields rigidity as well as a height difference of approximately $7 \mathrm{~nm}$ between the top and bottom (Figure 1a).

To realize a symmetric pattern of functionalization points, staple breaks are included regularly every two helical turns, creating a 7-nm-spaced rhombic pattern, with a total of 36 functional sites per disk face (Figure $1 \mathrm{a}$ and Figure S1). At these points, staples can be prolonged with single-stranded extensions, called "handles", which hybridize with their complementary sequence named "anti-handle". The substitution in the annealing mix of position-specific staples with the corresponding staple handle sequences enables the creation of geometrical patterns on the disk surface, available for functionalization with a biomolecule or imaging label of choice. A fully $\mathrm{H} / \mathrm{AH}$-functionalized structure with $2 \times 36$ regular 7-nm-spaced pixels can be created, which allows the presentation of a prescribed number of ligands at predetermined spacing with nanoscale precision.

The successful folding of the DNA origami disk was first assessed by agarose gel electrophoresis (Figure $1 \mathrm{~b}$ ) and TEM (Figure 1c and Figure S3). Next, we performed DNA-PAINT microscopy on a disk featuring a fully functionalized top surface with 36 handle sequences spaced approximately $7 \mathrm{~nm}$ apart. The bottom surface was modified with seven biotinylated staple strands (Figure S1) for stable surface attachment to a BSA-biotin-neutravidin functionalized glass slide. $^{22}$ The super-resolution reconstruction of single-disk structures showed well-resolved $7 \mathrm{~nm}$ patterns (Figure S4) with a sum image from 150 different structures, demonstrating the exquisite structural integrity and completeness of the DNA origami disk (Figure 1d).

In silico approximations of the distances between functionalization points yield $7.8 \mathrm{~nm}$ diagonal and $7.5 \mathrm{~nm}$ vertical point-to-point spacing (Figure 1a). A cross-sectional histogram analysis in the DNA-PAINT sum image confirms the average distances on the diagonal (i) and on the vertical line (ii) measured as $7.9 \pm 0.4$ and $7.7 \pm 0.2 \mathrm{~nm}$, respectively, closely matching the designed distances. Furthermore, we obtained a localization precision by nearest neighbor analysis (NeNA metric $)^{23}$ of $1.2 \mathrm{~nm}$, closely matching the measured localization precision in the sum images of $1.6 \mathrm{~nm}$ (obtained from the Gaussian fits to the sum image), yielding an achievable full width at half-maximum (fwhm)-limited resolution of $3.8 \mathrm{~nm}$ on these structures. This relatively high spatial resolution and close match of NeNA- and sum-image-based localization precisions underline the high formation yield and exquisite structural integrity of the DNA origami disk design. The latter is of paramount importance for spatiotemporal cellular interaction studies, where precise and accurate patterns of ligands are preferred. Therefore, our double-faced DNA origami disk constitutes a versatile nanomaterial platform for multivalent ligand presentation with accurate matching of pixel distances between in silico design and experimental analysis. A similar coherence between design and experiments has been obtained for functionalization patterns such as a large and small hexagon (Figure 1e and $\mathrm{f}$ ), a square (Figure $1 \mathrm{~g}$ ), and a triangle (Figure $1 \mathrm{~h}$ ), where $100-150$ origami were used to obtain the different sum images (see Figures S5-S8 for overview images). 

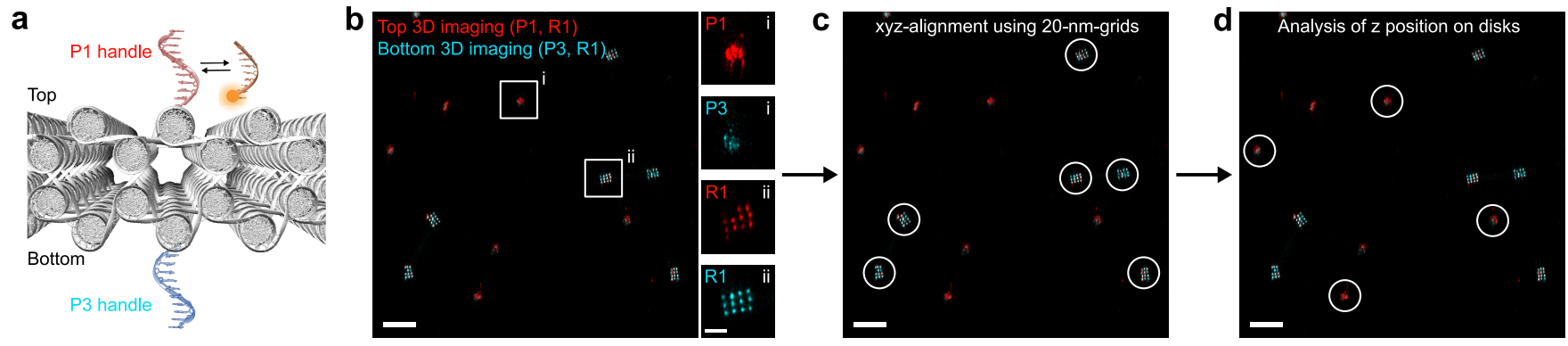

e

Determine $z$ position for top and bottom of disk
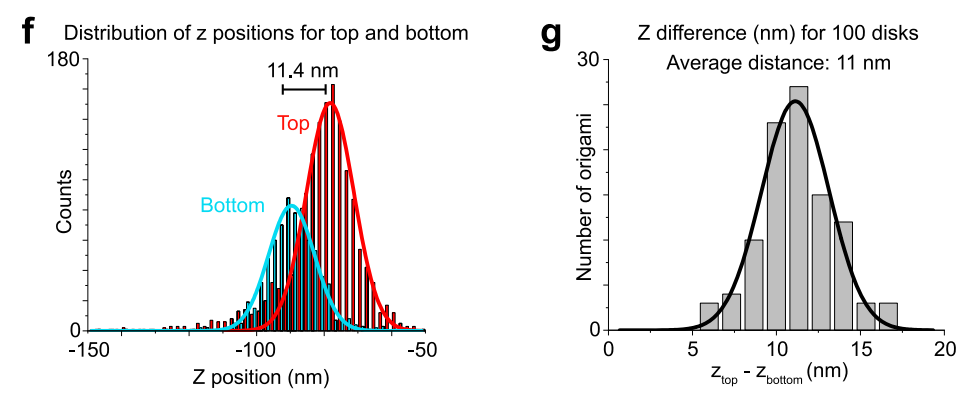

Figure 3. Precise disk top-to-bottom distance measurement using 3D DNA-PAINT. (a) 3D-rendered side view of the disk with top (red, P1 sequence) and bottom (cyan, P3 sequence) DNA-PAINT handles. (b) (Left) Two-round 3D Exchange-PAINT overview image of disks and $20 \mathrm{~nm}$ grids (red: first exchange round, cyan: second exchange round). (Right) Zoom-in of an exemplary disk (i) and $20 \mathrm{~nm}$ grid (ii) from both imaging rounds. (c) $20 \mathrm{~nm}$ grids are selected and used for precise 3D intraround drift correction and 3D inter-round alignment operating on single handle sites. (d, e) Multiple $7 \mathrm{~nm}$ disk patterns are selected for $3 \mathrm{D}$ analysis (d), and the $z$ positions of the respective top and bottom handles are separately pooled (e). (f) Histogram depicting the number of localizations plotted against their $z$ positions (in $\mathrm{nm}$ ) from both top (red) and bottom (cyan) sides of a single DNA origami disk. The corresponding Gaussian fits yield a peak-to-peak distance of $11.4 \mathrm{~nm}$. (g) Distribution of number of origami plotted against their peak-to-peak distances (in nm) for a total of 100 disks. The corresponding Gaussian fit yields an average top-to-bottom distance of $11 \pm 2 \mathrm{~nm}$. Scale bars: $200 \mathrm{~nm}$ (b overview, c, d), $50 \mathrm{~nm}(\mathrm{~b},(\mathrm{i})$ and (ii)).

Handle Accessibility and Hybridization Kinetics of Top and Bottom. Next, we turned our attention to the functionalization of both the top and bottom surface to assess potential differences in accessibility and DNA hybridization kinetics. The platform presents 36 pixels on each phase, designated "top" and "bottom" (Figure 2a). As described above, classical DNA-PAINT imaging experiments typically use DONs immobilized through binding of biotinylated handles protruding from the structure to a BSA-biotinneutravidin-modified glass surface. For flat structures, biotin handles are placed on the opposite side with respect to the docking handles. ${ }^{22}$ This effectively "sandwiches" modifications of the bottom surface of the DNA origami nanostructure between the core of the structure and the BSA-coated surface. Considering the respective size of neutravidin $(\sim 6 \mathrm{~nm})$ and BSA $(\sim 5 \mathrm{~nm})$ measured from the crystal structures (PDB 2AVI and 4F5S, respectively), we estimate the space between the DNA origami and the functionalized slide surface to be at least $10 \mathrm{~nm}$ (Figure 2a). This relatively tight space could potentially affect the accessibility of handle strands for hybridization of functional entities post-surface attachment (such as the binding of imager strands to docking strands in DNA-PAINT).

To assay potential differences in the accessibility of the space between the DNA origami and the functionalized slide surface, we decided to investigate three cases: (1) "dead zone", (2) "trapping zone" or (3) "active zone". In the case of a "dead zone", we would expect imager strands to be excluded from entering and imaging any patterns on a DON. For a "trapping zone", imager strands would be able to access the volume, but their association and dissociation kinetics might be significantly altered. In the case of an "active zone", no differences in imaging kinetics should be detectable.

To compare binding kinetics on the top and bottom of the disk, two different designs were used: one with a square pattern on the "top" side of the disk (and no pattern on the bottom) and another one with a triangle pattern on the "bottom" (and no pattern on the top). In both cases, the DNA-PAINT extensions generating the different patterns were designed with a docking site for the same imager sequence (called P3) for direct unbiased comparison (Figure 2a). Both "top square" and "bottom triangle" structures were present in the same sample, and DNA-PAINT acquisition was performed using the same imager strand. For the kinetic analysis, both structures were first selected using the "pick" tool in Picasso" ${ }^{19}$ (Figure 2b, white circles). Next, we applied our recently developed artificial neural network tool "nanoTRON" for shape identification (Figure 2c). ${ }^{24}$ First, we used separate DNAPAINT imaging data sets of either squares (Figure S9) or triangles (Figure S10) to train the software for pattern classification using the "Train" module. The training model for predicting squares or triangles was then saved and loaded into nanoTRON, and the module "Predict" was used to classify the origami in the data set of interest. This enabled us to distinguish the top squares and bottom triangles for downstream analysis (Figure 2d). Next, we selected single binding sites on the structures and analyzed the bright and dark times of these single spots for top and bottom squares separately (Figure $2 \mathrm{e}$ and $\mathrm{f}$ ). The bright time $\tau_{\mathrm{b}}$ (or "bound" time) corresponds to the time the imager strand is bound to its target docking strand, during which the imager's fluorophore can be detected by the camera. It is influenced by the length and DNA base composition of the imager strand. The dark time $\tau_{\mathrm{b}}$ (or 
a

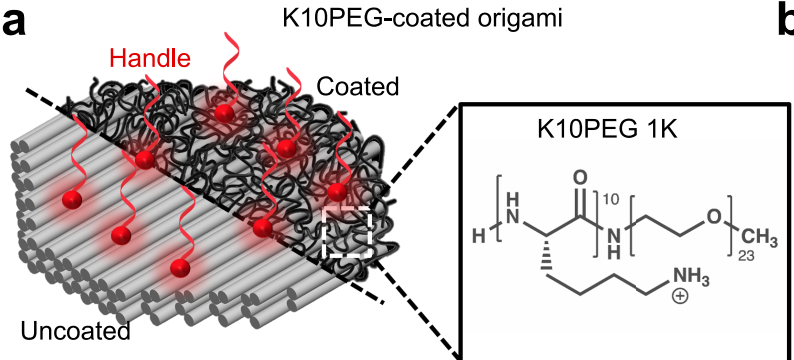

C

d

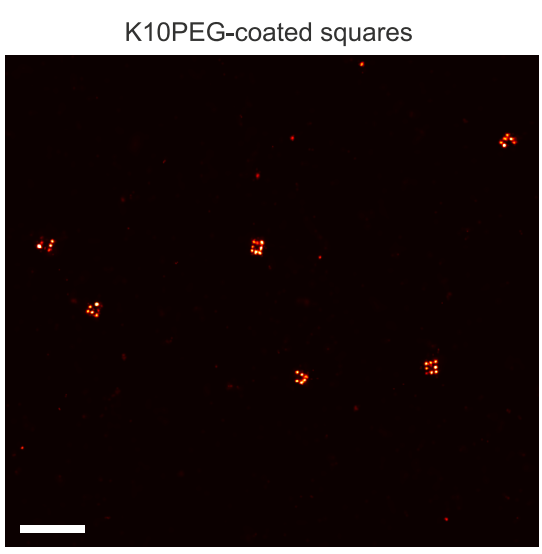

K10PEG-coated full pattern

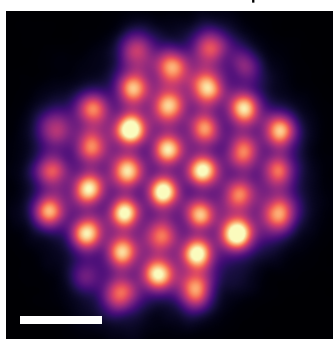

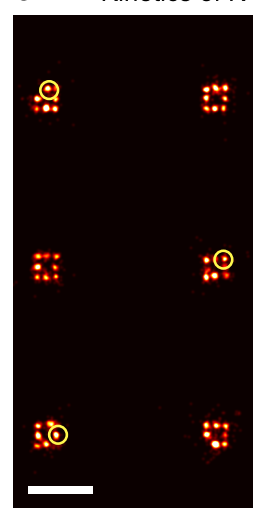

(1)
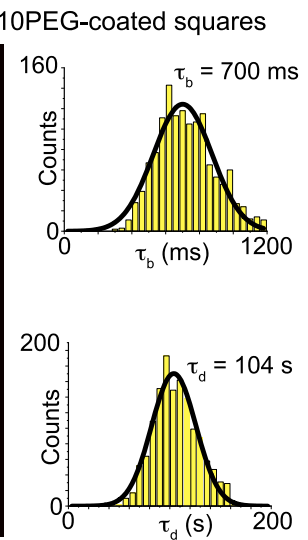

Figure 4. Accessibility and binding kinetics of K10PEG-protected structures. (a) Schematic representation of disk with a square pattern coated with K10PEG. The left side of the disk represents an uncoated disk. The right side of the disk represents a coated disk with a zoom-in of the chemical structure of the K10PEG 1K coating. (b) DNA-PAINT sum image of 150 K10PEG-coated full pattern disks with P1 imagers. The $7 \mathrm{~nm}$ spacing of the full pattern disk is still well resolved despite the K10PEG coating. (c) DNA-PAINT overview image of K10PEGcoated squares. (d) Assessment of hybridization kinetics to K10PEG-coated squares on the single-staple level. Bright time (top) and dark time (bottom) distributions and corresponding Gaussian fits yield average bright times of $673 \pm 68$ ms and average dark times of $107 \pm 5$ ms. The experiment was repeated three times independently with similar results. Scale bars: $15 \mathrm{~nm}(\mathrm{~b}), 200 \mathrm{~nm}(\mathrm{c})$, and $100 \mathrm{~nm}(\mathrm{~d})$.

"dissociated" time) corresponds to the dwell time of the unbound state, during which no imager strand is bound to the same target. It can be adjusted by varying the imager or salt concentration and, to some extent, the sequence. ${ }^{25,26}$

The analysis surprisingly revealed that both patterns on the top and bottom exhibit closely matching binding kinetics (see Figures S11 and S12 for an overview of origami used for kinetic calculations, Table S1 for kinetic summary and Figure S13 for comparison of top triangle and top square patterns as a control). These findings suggest that despite the limited space of $\sim 10 \mathrm{~nm}$ between the glass and the surface of the DON, the eight-nucleotide-long imager strands (estimated 2-3 $\mathrm{nm}$ in diameter) has sufficient space to enter and leave this volume without affecting the binding kinetics. Therefore, the volume beneath a DON can be confidently labeled as "active" and well accessible, presenting no significant differences compared to imaging in the "open space" from above the structure.

Direct 3D Visualization of Top and Bottom Surfaces. Next, we designed a 3D DNA-PAINT imaging experiment to directly assess if handle extensions designed to protrude from the top and bottom are indeed positioned as expected in 3D space on both sides of the structure. As earlier reports for single-layer DNA origami structures have suggested the possible threading of handle sequences and modifications "through" the sheets, ${ }^{27}$ we set out to probe this for our DNA origami disk structure. While DNA-PAINT's $1.2 \mathrm{~nm}$ localization precision in our $2 \mathrm{D}$ experiments enabled us to resolve the $7 \mathrm{~nm}$ spacing of the handle sequences with relative ease, localization precision and thus resolution along the microscope's optical axis in $z$ are typically more than 2 -fold worse for astigmatism-based 3D super-resolution approaches. ${ }^{28,29} \mathrm{We}$ expect the distance between the structure's top and bottom to be in a similar $\sim 6-12 \mathrm{~nm}$ distance range as the $2 \mathrm{D}$ handle-tohandle spacing per DNA origami design taking previously reported interhelical gaps into account. ${ }^{30,31}$ Since resolving 6$12 \mathrm{~nm}$ distances in $z$ using a single imager and docking sequence in a single round of imaging will be challenging, we here opted to average localization from the respective top and bottom surface using two rounds of Exchange-PAINT imaging. ${ }^{19,32}$ By temporally separating top and bottom surfaces of the structures, we could faithfully assign localizations to the top and bottom surface, respectively, for precise distance measurement. To achieve this, the disk was extended with P1 docking handles for the top surface (Figure 3a, represented by one red strand) and P3 docking handles for the bottom side (Figure $3 a$, represented by one blue strand). We then performed two rounds of 3D Exchange-PAINT and determined the distance in $z$ between the P1 handles on the top and the P3 handles on the bottom.

To ensure measurements were performed in a robust, driftand alignment-unbiased fashion, we added 20-nm-grid singlelayered DNA origami structures ${ }^{25}$ to the same sample containing the DNA origami disks. To guarantee that the 20-nm-grid structures were imaged independently in each Exchange-PAINT round, we used speed-improved handle sequences $^{25}$ and corresponding imager strands (present in both exchange rounds) for the grid structures (Figure $3 b$ ). Post-Exchange-PAINT acquisition, we used the $20 \mathrm{~nm}$ grids for intraround drift correction and accurate inter-round alignment of all single handle positions in $x, y$, and $z$. This 
could be achieved in a robust and unbiased fashion, since the complementary imager strands to the $\mathrm{R} 1$ handles on the 20 $\mathrm{nm}$ - rids could only bind to the top side of the origami, basically representing prescribed single-molecule fiducial points in $x y z$-space (Figure 3c). After drift correction and alignment, single disk structures were selected for further analysis (Figure 3d).

To calculate the distance between top and bottom handles on the structures, we carried out the following analysis for each structure individually: We created a cross-sectional histogram in the $x y$-plane (essentially plotting the distribution of all measured $z$ positions per structure) for the top (P1) and bottom (P3) acquisition separately (Figure 3e), fitted each distribution with a Gaussian function, and calculated the peakto-peak distance on a per-structure basis (Figure 3f). We then performed this analysis for 100 disk structures separately and plotted a histogram for the peak-to-peak distances from all structures (Figure 3g, see Figure S14 for overview of selected disks). We obtained an average top-to-bottom distance of 11 $\mathrm{nm}$ with a standard deviation of $2 \mathrm{~nm}$. This not only confirmed a precise and accurate placement of handles on both the top and bottom surface of the disk, but the very high $z$-resolution obtained through Exchange-PAINT (in this case a localization precision of $1.4 \mathrm{~nm}$, suggesting an fwhm-limited $z$-resolution of $3.3 \mathrm{~nm}$ ) allowed us to estimate an average helix diameter in this DNA origami design based on a honeycomb geometry of $2.6 \mathrm{~nm}$ (assuming a $0.63 \mathrm{~nm}$ distance per base in ssDNA ${ }^{33}$ and taking into account that $2 \mathrm{~T}$ spacers were added to handle extensions on the top and bottom surface), which is in exquisite agreement with earlier studies based on CryoEM ${ }^{30}$ and SAXS. ${ }^{31}$

Accessibility of Handles in Coated Structures. Finally, we turned our attention to evaluating accessibility and hybridization kinetics of K10PEG-protected DNA origami disk structures. The first hurdles to transfer DNA nanotechnology into biological applications are overcome by solving the stability challenges in the form of applying a gentle coating strategy, ${ }^{17}$ which ensures the integrity of DONs also in physiological conditions presenting critical low levels of $\mathrm{Mg}^{2+}$ concentration and high concentration of nucleases. While performing its protective role, a downside exists in the potential overall occlusion of functionalities added to the DON for controlled cell interaction and manipulation. It is therefore imperative to ensure that the coating will not hinder the accessibility of ligands on the surface of DONs. As a final set of experiments in this study, we therefore coated our disks following the K10PEG coating protocol ${ }^{17}$ using a $1: 1 \mathrm{~N}: \mathrm{P}$ ratio, as previously optimized for biostability studies. ${ }^{17}$ First, we qualitatively tested handle accessibility by gel electrophoresis where dye-labeled anti-handles were incubated with the K10PEG-coated origami. The gel revealed that handles were still accessible after K10PEG coating (Figure S15). We then repeated the super-resolution imaging of the full $7 \mathrm{~nm}$ pattern and square pattern on the top sides of the disk (Figure 4a). Surprisingly, the $7 \mathrm{~nm}$ pattern could still be clearly resolved after K10PEG coating (Figure 4b, Figure S16) with a similar NeNA-based localization precision of $1.8 \mathrm{~nm}$ compared to uncoated structures. However, the coating had some effects on the DNA-PAINT imaging of the structures since a higher concentration of origami ( $\sim 10$-fold) was required in order to obtain a similar number of DONs in the field of view as for the uncoated structures. Expectedly, the K10PEG coating seems to cover the biotinylated staples that are integrated into the core structure and not protruding through handles, thereby significantly hindering origami attachment to the functionalized glass slide. For those attached, the gap between the K10PEG meshwork was sufficient to enable origami binding. K10PEG-coated squares were also easily recognizable (Figure 4c) and were used for downstream binding kinetic evaluation of K10PEG-coated structures. Interestingly, the binding kinetics measured on the K10PEG-coated squares (Figure $4 \mathrm{~d}$, see Figure $\mathrm{S} 17$ for overview of origami used for kinetic calculations) were indistinguishable from uncoated squares (Figure 2e and Table S1), confirming that the K10PEG meshwork leaves DNA handles still accessible for downstream modification by hybridization. This can be explained by the fact that the docking strands sticking out of the origami are $\sim 7$ $\mathrm{nm}$ in length (when fully extended), whereas the K10PEG coating only covers the origami with an estimated $1 \mathrm{~nm}$ in thickness, leaving space for the imager strands to bind. In addition, it is possible that the K10PEG meshwork is flexible enough to allow imager strands binding to their docking strands.

\section{CONCLUSIONS}

In this study, we introduce a DNA origami disk design featuring $2 \times 36$ regularly spaced attachment points on both faces of the structure, poised as a universal platform for cellular interaction studies on the true level of single biomolecules. We employ DNA-PAINT super-resolution microscopy on the level of single handle strands with sub-3 $\mathrm{nm}$ spatial resolution to precisely characterize the integrity and addressability of the DNA origami disk and find the design to be structurally precise on the one-nanometer level. This is an important prerequisite for its application as a precision scaffold for the arrangement of ligands and biomolecules in biological studies. In particular, nanoscale accuracy in ligand presentation is fundamental for biomedical targets where spatial organization of proteins is known to moderate signaling, for instance in the immune synapse $^{34}$ or focal adhesion formation. ${ }^{35}$ Even when linked to a BSA-biotin-neutravidin surface, both the top and bottom of the DNA origami disk are still addressable and handle extensions are available for DNA-PAINT microscopy. Interestingly binding kinetic measurements on the single-handle level show no difference between the top and bottom face of the structure, which is of paramount importance for downstream applications of the DNA origami disk as an interfacing entity for cell attachment. Our findings imply that both sides of the structure can be used in a similar fashion for functionalization and downstream visualization in, for example, barcoded fluorescence imaging studies, as the neutravidin used for surface attachment represents a good model protein regarding size for future entities to be attached to the structures.

Using high-resolution 3D Exchange-PAINT, we were able to clearly visualize the top and bottom of the structure and determined a distance of $11 \mathrm{~nm}$ between the strands on the two faces of the DNA origami disk. Again, we find the structure design to be precise also for top and bottom attachment of functional entities. The high $z$-resolution enabled us to calculate the apparent diameter of DNA double helices from 3D DNA-PAINT measurements to be $2.6 \mathrm{~nm}$, in excellent agreement with earlier studies employing CryoEM ${ }^{30}$ and SAXS. ${ }^{31}$

Finally, the application of a protective coating has enabled the initial translational steps and dreams to translate the DNA origami technology toward nanotherapeutic targets. We 
therefore investigated the impact of protective coating of DNA origami structure on the downstream accessibility and functionalization of the structure. While the coating protects the structural integrity of the DON, it could have negative effects on the protrusion efficacy of functional sites, often used to include targeting moieties or activation markers. Excitingly, our results show that for an N:P 1:1 coating with K10PEG the handles are still accessible. Even more surprisingly, binding kinetics in DNA-PAINT experiments are unaltered compared to uncoated structures. Taken together, the insights presented in our results bring this DNA-based materials platform one step closer to applications in cellular targeting, diagnostics, and therapeutic drug delivery.

\section{METHODS}

Materials. Unmodified DNA oligonucleotides were purchased from Integrated DNA Technologies. Cy3B-modified DNA oligonucleotides were ordered from MWG Eurofins. Scaffolds type p7560 and type p7249 (M13mp18) were obtained from Tilibit (cat: M1-30 and M1-10, respectively). Magnesium 1 M (cat: AM9530G), sodium chloride $5 \mathrm{M}$ (cat: AM9759), ultrapure water (cat: 10977035), Tris 1 M, pH 8.0 (cat: AM9855G), EDTA 0.5 M, pH 8.0 (cat: AM9260G), $10 \times$ PBS (cat: 70011051), SYBR Safe (cat: S33102), glass slides (cat: 10756991), and neutravidin (cat: 3100) were purchased from Thermo Fisher Scientific. Ladder $1 \mathrm{kB}$ (N3232L) was purchased from Biolabs. Potassium chloride (cat: 6781.1) and agarose (cat: 3810.2) were purchased from Carl Roth. Gel loading dye (cat: B7024S) was purchased from New England Biolabs. Sodium hydroxide $2 \mathrm{M}$ (cat: 1091361000) was purchased from Merck. Tween 20 (cat: P9416-50 ML), protocatechuate 3,4-dioxygenase pseudomonas (PCD) (cat: P8279), 3,4-dihydroxybenzoic acid (PCA) (cat: 37580-25G-F), glycerol (65516-500 mL), (+-)-6-hydroxy2,5,7,8-tetra-methylchromane-2-carboxylic acid (Trolox) (cat: 238813-5 G), methanol (cat: 32213-2.5L), and BSA-biotin (cat: A8549) were ordered from Sigma-Aldrich. Oligolysine-PEG $1 \mathrm{~K}$ (mPEG1K-b-PLKC10) was purchased from Alamanda Polymers. Large coverslips (cat: 0107242) were purchased from Marienfeld, and $90 \mathrm{~nm}$ diameter gold nanoparticles (cat: G-90-100) were ordered from Cytodiagnostics.

Buffers. The following buffers were used for sample preparation and DNA-PAINT imaging: buffer A: $10 \mathrm{mM}$ Tris $\mathrm{pH} 8.0,100 \mathrm{mM}$ $\mathrm{NaCl}, 0.05 \%$ Tween 20; buffer B: $5 \mathrm{mM}$ Tris- $\mathrm{HCl} \mathrm{pH} 8,10 \mathrm{mM}$ $\mathrm{MgCl}_{2}, 1 \mathrm{mM}$ EDTA, $0.05 \%$ Tween 20, $\mathrm{pH}$ 8.0; imaging buffer: buffer $B$ supplemented with $1 \times$ Trolox, $1 \times$ PCA and $1 \times$ PCD; buffer C: $1 \times$ PBS, $1 \mathrm{mM}$ EDTA, $500 \mathrm{mM} \mathrm{NaCl}, \mathrm{pH}$ 7.4; disk folding buffer $(1 \times)$ : $5 \mathrm{mM}$ Tris, $1 \mathrm{mM}$ EDTA, $5 \mathrm{mM} \mathrm{NaCl}, 20 \mathrm{mM} \mathrm{MgCl}$, $\mathrm{pH} 8.0$; 20$\mathrm{nm}$-grid folding buffer $(1 \times): 10 \mathrm{mM}$ Tris, $1 \mathrm{mM}$ EDTA, $12.5 \mathrm{mM}$ $\mathrm{MgCl}_{2}, \mathrm{pH}$ 8.0; PCA, PCD, and Trolox, 40× PCA: $154 \mathrm{mg}$ PCA was dissolved in $10 \mathrm{~mL}$ of $\mathrm{H}_{2} \mathrm{O}$ and adjusted to $\mathrm{pH} 9.0$ using $\mathrm{NaOH}$, 100X PCD: $9.3 \mathrm{mg}$ PCD was dissolved in $13.3 \mathrm{~mL}$ of buffer $(100 \mathrm{mM}$ Tris-HCl pH 8, $50 \mathrm{mM} \mathrm{KCl,} 1 \mathrm{mM}$ EDTA, 50\% glycerol), 100× Trolox: $100 \mathrm{mg}$ of Trolox was dissolved in $3.2 \mathrm{~mL}$ of $\mathrm{H}_{2} \mathrm{O}$ complemented with $430 \mu \mathrm{L}$ of $100 \%$ methanol and $345 \mu \mathrm{L}$ of $1 \mathrm{M}$ $\mathrm{NaOH}$.

DNA Origami Self-Assembly. The DNA origami disk structure was designed with the software cadnano. ${ }^{36}$ An initial folding test was performed as previously described ${ }^{37}$ to assess optimal folding conditions (Figure S18). Self-assembly of the DNA origami disk was accomplished in a one-pot reaction mix with $50 \mu \mathrm{L}$ total volume, consisting of $20 \mathrm{nM}$ scaffold strand p7560 (sequence see Table S2), $200 \mathrm{nM}$ core folding staples (Table S3), $200 \mathrm{nM}$ staple sequences without handle extension (Table S4), $500 \mathrm{nM}$ biotinylated staples (Table S5), and $1 \mu \mathrm{M}$ of staple strands with docking site extensions (Tables S6) in $1 \times$ disk folding buffer. For each disk design, when staple strands with docking site extensions are used, the corresponding staples without a handle extension need to be removed from the mix (see Tables S7 and S8 for staples of different patterns). The reaction mix was then subjected to a thermal annealing ramp using a thermocycler (Eppendorf, cat: 6331000017). The reaction mix was first incubated at $80{ }^{\circ} \mathrm{C}$ for $5 \mathrm{~min}$, then cooled using a temperature gradient from $60{ }^{\circ} \mathrm{C}$ to $20^{\circ} \mathrm{C}$ in steps of $-1{ }^{\circ} \mathrm{C}$ per hour and finally held at $20^{\circ} \mathrm{C}$. Self-assembly of the $20 \mathrm{~nm}$ grid was performed in a one-pot reaction mix with $40 \mu \mathrm{L}$ total volume, consisting of $10 \mathrm{nM}$ scaffold strand p7249 (M13mp18, Table S9) with $100 \mathrm{nM}$ folding staples, $500 \mathrm{nM}$ biotinylated staples, and $1 \mu \mathrm{M}$ staple strands with docking site extensions (all staples are listed in Table S10) in $1 \times 20 \mathrm{~nm}$ grid folding buffer. The reaction mix was then subjected to a thermal annealing ramp using a thermocycler. The reaction mix was first incubated at $80{ }^{\circ} \mathrm{C}$ for $5 \mathrm{~min}$, then cooled using a temperature gradient from $60{ }^{\circ} \mathrm{C}$ to $4{ }^{\circ} \mathrm{C}$ in steps of $1{ }^{\circ} \mathrm{C}$ per 3.21 min and finally held at $4{ }^{\circ} \mathrm{C}$.

DNA Origami Purification. The disk-shaped DNA origami structures were gel-purified by mixing $25 \mu \mathrm{L}$ of origami with $5 \mu \mathrm{L}$ of $6 \times$ loading dye and subsequently subjected to agarose gel electrophoresis ( $1.5 \%$ agarose, $\left.1 \times \mathrm{TA}, 10 \mathrm{mM} \mathrm{MgCl}_{2}, 1 \times \mathrm{SYBR}_{\text {Safe }}\right)$ at 77 $\mathrm{V}$ for $1.5 \mathrm{~h}$ in a $4{ }^{\circ} \mathrm{C}$ room. Gel bands were extracted and crushed. Agarose gel purification of the disk-shaped origami is mainly performed to remove the excess of staple strands with DNA-PAINT docking sites or the biotinylated staple strands that could increase the background noise during the imaging experiments. The $20 \mathrm{~nm}$ grids were purified via ultrafiltration using Amicon Ultra centrifugal filters with a $50 \mathrm{kDa}$ molecular weight cutoff (MWCO; Merck Millipore, cat: UF505096) as previously described. ${ }^{37}$ The columns were first equilibrated with $500 \mu \mathrm{L}$ of buffer $\mathrm{C}$ and centrifuged (Eppendorf, cat: $5404000537)$ at $7500 \mathrm{~g}$ for $10 \mathrm{~min}$. Then, the folded origami were brought to $500 \mu \mathrm{L}$ with buffer $\mathrm{C}$, added to the filters, and centrifuged for $5 \mathrm{~min}$ at $5000 \mathrm{~g}$. This process was repeated twice. Purified origami were recovered into a new tube by centrifugation for $5 \mathrm{~min}$ at $5000 \mathrm{~g}$. After purifying the origami using either method, the resulting concentration was measured by absorbance at $260 \mathrm{~nm}$ using a Nanodrop (Thermo Fisher Scientific, cat: ND-ONE-W), and the purified structures were kept at $-20{ }^{\circ} \mathrm{C}$.

Analytical Agarose Gels. To assess the quality of the origami folding and purification, $10 \mu \mathrm{L}$ of annealing mix and gel-purified DNA origami structures were mixed with $2 \mu \mathrm{L}$ of $6 \times$ loading dye and subsequently subjected to agarose gel electrophoresis ( $1 \%$ agarose, $1 \times$ TBE, $20 \mathrm{mM} \mathrm{MgCl}_{2}, 1 \times$ SYBR Safe) at $70 \mathrm{~V}$ for $1.5 \mathrm{~h}$ in an ice-water bath. The gels were imaged using a BioRad ChemiDoc MP (cat: 17001402).

TEM Imaging. DNA origami annealing mix was purified, and $3 \mu \mathrm{L}$ of samples at $20 \mathrm{nM}$ concentration was adsorbed on a carbon grid and incubated for $2 \mathrm{~min}$. The excess solution was removed by blotting the grid on Whatman paper and immediately adding $5 \mu \mathrm{L}$ of $2 \%$ uranyl formate on top of the grid for $30 \mathrm{~s}$. Finally, the excess solution was removed with Whatman paper and the grid was left drying for at least $10 \mathrm{~min}$. Samples were imaged on a Tecnai T12 transmission electron microscope operated at a voltage of $100 \mathrm{kV}$ with a magnification of $3810000 \times$.

DNA-PAINT Sample Preparation. All DNA-PAINT experiments were performed using ibidi channels with glass bottoms (ibidi, 80607 ). First, $100 \mu \mathrm{L}$ of biotin-labeled bovine albumin (BSA-biotin, 1 $\mathrm{mg} / \mathrm{mL}$, dissolved in buffer A) was flushed into the channel and incubated for $5 \mathrm{~min}$. The channel was then washed with $1 \mathrm{~mL}$ of buffer A. A volume of $100 \mu \mathrm{L}$ of neutravidin $(0.1 \mathrm{mg} / \mathrm{mL}$, dissolved in buffer A) was then flushed through the channel and allowed to bind for $5 \mathrm{~min}$. After washing with $1 \mathrm{~mL}$ of buffer $\mathrm{A}$ and subsequently with $1 \mathrm{~mL}$ of buffer B, $70 \mu \mathrm{L}$ of biotin-labeled DNA structures $(\sim 200 \mathrm{pM})$ in buffer B was flushed into the chamber and incubated for $15 \mathrm{~min}$. The chamber was then washed with $1 \mathrm{~mL}$ of buffer B. After origami incubation, $150 \mu \mathrm{L}$ of gold nanoparticles (diluted 1:10 in buffer $\mathrm{C}$ ) was flushed through and incubated for $5 \mathrm{~min}$. After washing with buffer $\mathrm{B}, 500 \mu \mathrm{L}$ of imagers diluted in the imaging buffer was flushed into the chamber (see Table S11 for imager and docking sequences). In between imaging rounds of multiplexing experiments, the sample was washed 4 or 5 times with buffer B until no residual signal from the previous imager solution was detected. The next imager solution was then introduced. 
K10PEG Coating for DNA-PAINT Imaging. To coat the DNA origami, purified origami stock was diluted to $1 \mathrm{nM}$ final concentration in $1 \times$ disk folding buffer to a final volume of $25 \mu \mathrm{L}$. It was then mixed with a solution of K10PEG $(1 \mathrm{~K})$ of equal volume $(25 \mu \mathrm{L})$ to obtain a final ratio of nitrogens in lysines of the coating $v s$ phosphates of DNA (N:P ratio) of 1:1, as previously described. ${ }^{17}$ The origami was then flushed through an ibidi channel (preemptively prepared with BSA-biotin and neutravidin), and the addition of gold and imager solution was performed following the same sample preparation described above.

K10PEG Coating for Gel Analysis. To coat the DNA origami, 10 $\mu \mathrm{L}$ of purified origami stock at $10 \mathrm{nM}$ in $1 \times$ folding buffer was mixed with a solution of K10PEG (1K) of equal volume to obtain a final ratio of nitrogens in lysines of the coating $v s$ phosphates of DNA $(\mathrm{N}: \mathrm{P}$ ratio) of $1: 1$, as previously described. ${ }^{7}$ After incubation at room temperature for $30 \mathrm{~min}, 10 \mu \mathrm{L}$ of sample was mixed with $2 \mu \mathrm{L}$ of $6 \times$ loading dye and subsequently subjected to agarose gel electrophoresis ( $1 \%$ agarose, $1 \times \mathrm{TBE}, 20 \mathrm{mM} \mathrm{MgCl}{ }_{2}, 1 \times \mathrm{SYBR}$ Safe) at $70 \mathrm{~V}$ for 1.5 $\mathrm{h}$ in an ice-water bath. The gels were imaged using BioRad ChemiDoc MP (cat: 17001402). Successful coating can be assessed since coated samples are not migrating in agarose gel electrophoresis but remain in the well pocket.

Microscope Setup. Imaging was carried out using an inverted microscope (Nikon Instruments, Eclipse Ti2) and the Perfect Focus System, by applying an objective-type total internal reflection fluorescence (TIRF) configuration with an oil-immersion objective (Nikon Instruments, Apo SR TIRF 100×, NA 1.49, oil). A $561 \mathrm{~nm}$ laser (MPB Communications Inc., $500 \mathrm{~mW}$, DPSS-system) was used for excitation and was coupled into a single-mode fiber. The laser beam was passed through cleanup filters (Chroma Technology, ZET561/10) and coupled into the microscope objective using a beam splitter (Chroma Technology, ZT561rdc). Fluorescence light was spectrally filtered with an emission filter (Chroma Technology, ET600/50m and ET575lp) and imaged with an sCMOS camera (Andor, Zyla 4.2 Plus) without further magnification, resulting in an effective pixel size of $130 \mathrm{~nm}$ after $2 \times 2$ binning. Camera readout sensitivity was set to 16-bit, and readout bandwidth to $540 \mathrm{MHz}$. Imaging parameters used in the different experiments are shown in Table S12, and NeNA values are listed in Table S13.

Image Analysis. Raw fluorescence data were subjected to superresolution reconstruction using the Picasso software package ${ }^{19}$ (latest version available at https://github.com/jungmannlab/picasso). Drift correction was performed with a redundant cross-correlation and gold nanoparticles as fiducials in Picasso Localize. Prior to kinetic analysis, localizations were linked allowing a gap size of 3 frames and a maximum diameter of 0.5 pixel. Kinetic information on detected picks was extracted with the Save pick properties command in Picasso Render. Further quantification such as histogram analysis and fitting was performed using Origin Pro 2019b (OriginLab Corporation).

NanoTRON Analysis. Two training data sets were generated: one with only squares and one with only triangles. More than 500 origami were picked per training data using the Save picked localizations in Picasso Render. In the nanoTRON module Train Model, the Number of Classes was set to 2. The two Training Files were uploaded and were assigned the class names "Triangle1" and "Square1". Expanding training data set was selected, but the other nanoTRON default settings were kept (Oversampling: 50, Hidden Layers: 1, Nodes: 500, Solver: adam, Activation: relu, Iterations: 400, Learning rate: 0.0010). ${ }^{24}$ Training was started by selecting the button Train. The resulting model was subsequently saved and had a Test Accuracy of 0.99 with a Train Loss of 0.01 for a Train Accuracy of 1.0. For structure prediction, the saved model was imported via Load Model and the Filter Probabilities was set to 0.99 . The data set of interest containing both picked squares and triangles was then uploaded, the options Export Pick Regions and Regroup Export Files were selected, and the prediction started by selecting the button Predict. After the prediction finished, the classified nanopatterns were exported in separate files by selecting the button Export. The exported separated triangles and squares were then subject to further kinetic analysis as described above.

\section{ASSOCIATED CONTENT}

\section{Supporting Information}

The Supporting Information is available free of charge at https://pubs.acs.org/doi/10.1021/acsnano.1c05540.

Detailed DNA origami design and characterization section: caDNAno file overview; $3 \mathrm{D}$ rendering of DNA origami disk; additional field of view for TEM images; agarose gel electrophoresis of coated disks and assessment of handle accessibility; extended super-resolution data: DNA-PAINT overview images; binding kinetics; initial folding test of DNA origami disk; summary of binding kinetics; sequences for DNA origami and imager strands; imaging parameters and calculated NeNA values (PDF)

\section{AUTHOR INFORMATION}

\section{Corresponding Authors}

Ralf Jungmann - Faculty of Physics and Center for Nanoscience, Ludwig Maximilian University, 80539 Munich, Germany; Max Planck Institute of Biochemistry, Martinsried 82152, Germany; 이이이.org/0000-0003-4607-3312; Email: jungmann@biochem.mpg.de

Maartje M. C. Bastings - Programmable Biomaterials Laboratory, Institute of Materials, School of Engineering, Ecole Polytechnique Fédérale Lausanne, Lausanne 1015, Switzerland; Interfaculty Bioengineering Institute, School of Engineering, Ecole Polytechnique Fédérale Lausanne, Lausanne 1015, Switzerland; 이이. orcid.org/000-00027603-4018; Email: maartje.bastings@epfl.ch

\section{Authors}

Alexandra S. Eklund - Faculty of Physics and Center for Nanoscience, Ludwig Maximilian University, 80539 Munich, Germany; Max Planck Institute of Biochemistry, Martinsried 82152, Germany; (i) orcid.org/0000-0003-1047-2100

Alice Comberlato - Programmable Biomaterials Laboratory, Institute of Materials, School of Engineering, Ecole Polytechnique Fédérale Lausanne, Lausanne 1015, Switzerland

Ian A. Parish - Peter MacCallum Cancer Centre, Melbourne, VIC 3000, Australia; Sir Peter MacCallum Department of Oncology, University of Melbourne, Melbourne, VIC 3128, Australia

Complete contact information is available at: https://pubs.acs.org/10.1021/acsnano.1c05540

\section{Author Contributions}

${ }^{\#}$ A.S.E. and A.C. contributed equally.

\section{Author Contributions}

A.S.E. performed DNA-PAINT imaging experiments, analyzed data, and contributed to the writing of the manuscript. A.C. designed the DNA disk, optimized folding conditions and stability assays, analyzed data, and contributed to the writing of the manuscript. I.A.P. conceived the study and contributed to critical discussions during the design process and biological context. M.M.C.B. and R.J. conceived and supervised the study, interpreted data, and wrote the manuscript. All authors reviewed and approved the manuscript.

\section{Funding}

This work has been supported by the Human Frontier Science Program (HFSP) through the Young Investigator Grant RGY0065/2018 and in part by the German Research 
Foundation through the SFB1032 (Project-ID 201269156, A11, R.J.), the European Research Council through an ERC Starting Grant (MolMap; grant agreement number 680241, R.J.), the Max Planck Society (R.J.), the Danish National Research Foundation (Centre for Cellular Signal Patterns, DNRF135, R.J. and A.S.E.), and the "Fondation Pierre Mercier pour la Science” (M.M.C.B. and A.C.).

\section{Notes}

The authors declare no competing financial interest.

\section{ACKNOWLEDGMENTS}

We thank Sayed Ali Khoshouei for TEM images, Fabian Schneider for help with the initial folding test for the DNA origami disk, and Thomas Schlichthaerle for support with data analysis. A.S.E. acknowledges the International Max Planck Research School for Molecular and Cellular Life Sciences (IMPRS-LS).

\section{REFERENCES}

(1) Seeman, N. C.; Sleiman, H. F. DNA Nanotechnology. Nat. Rev. Mater. 2018, 3 (1), DOI: 10.1038/natrevmats.2017.68.

(2) Praetorius, F.; Kick, B.; Behler, K. L.; Honemann, M. N.; Weuster-Botz, D.; Dietz, H. Biotechnological Mass Production of DNA Origami. Nature 2017, 552 (7683), 84-87.

(3) Bila, H.; Kurisinkal, E. E.; Bastings, M. M. C. Engineering a Stable Future for DNA-Origami as a Biomaterial. Biomater. Sci. 2019, 7 (2), 532-541.

(4) Li, J.; Fan, C.; Pei, H.; Shi, J.; Huang, Q. Smart Drug Delivery Nanocarriers with Self-Assembled DNA Nanostructures. Adv. Mater. 2013, 25 (32), 4386-96.

(5) Liu, S.; Jiang, Q.; Zhao, X.; Zhao, R.; Wang, Y.; Wang, Y.; Liu, J.; Shang, Y.; Zhao, S.; Wu, T.; Zhang, Y.; Nie, G.; Ding, B. A DNA Nanodevice-Based Vaccine for Cancer Immunotherapy. Nat. Mater. 2021, 20 (3), 421-430.

(6) Yang, Y. R.; Liu, Y.; Yan, H. DNA Nanostructures as Programmable Biomolecular Scaffolds. Bioconjugate Chem. 2015, 26 (8), 1381-95.

(7) Rinker, S.; Ke, Y.; Liu, Y.; Chhabra, R.; Yan, H. Self-Assembled DNA Nanostructures for Distance-Dependent Multivalent LigandProtein Binding. Nat. Nanotechnol. 2008, 3 (7), 418-22.

(8) Wagenbauer, K. F.; Wachauf, C. H.; Dietz, H. Quantifying Quality in DNA Self-Assembly. Nat. Commun. 2014, 5, 3691.

(9) Myhrvold, C.; Baym, M.; Hanikel, N.; Ong, L. L.; Gootenberg, J. S.; Yin, P. Barcode Extension for Analysis and Reconstruction of Structures. Nat. Commun. 2017, 8, 14698.

(10) Strauss, M. T.; Schueder, F.; Haas, D.; Nickels, P. C.; Jungmann, R. Quantifying Absolute Addressability in DNA Origami with Molecular Resolution. Nat. Commun. 2018, 9 (1), 1600.

(11) Fang, T.; Alvelid, J.; Spratt, J.; Ambrosetti, E.; Testa, I.; Teixeira, A. I. Spatial Regulation of T-Cell Signaling by Programmed Death-Ligand 1 on Wireframe DNA Origami Flat Sheets. ACS Nano 2021, 15 (2), 3441-3452.

(12) Voigt, N. V.; Torring, T.; Rotaru, A.; Jacobsen, M. F.; Ravnsbaek, J. B.; Subramani, R.; Mamdouh, W.; Kjems, J.; Mokhir, A.; Besenbacher, F.; Gothelf, K. V. Single-Molecule Chemical Reactions on DNA Origami. Nat. Nanotechnol. 2010, 5 (3), 200-3.

(13) Wang, P.; Rahman, M. A.; Zhao, Z.; Weiss, K.; Zhang, C.; Chen, Z.; Hurwitz, S. J.; Chen, Z. G.; Shin, D. M.; Ke, Y. Visualization of the Cellular Uptake and Trafficking of DNA Origami Nanostructures in Cancer Cells. J. Am. Chem. Soc. 2018, 140 (7), $2478-2484$.

(14) Green, C. M.; Hughes, W. L.; Graugnard, E.; Kuang, W. Correlative Super-Resolution and Atomic Force Microscopy of DNA Nanostructures and Characterization of Addressable Site Defects. ACS Nano 2021, 15, 11597.

(15) Tsunoda, S.; Sierralta, J.; Sun, Y.; Bodner, R.; Suzuki, E.; Becker, A.; Socolich, M.; Zuker, C. S. A Multivalent PDZ-Domain
Protein Assembles Signalling Complexes in a G-Protein-Coupled Cascade. Nature 1997, 388 (6639), 243-9.

(16) Sriram, S. M.; Banerjee, R.; Kane, R. S.; Kwon, Y. T. Multivalency-Assisted Control of Intracellular Signaling Pathways: Application for Ubiquitin- Dependent N-End Rule Pathway. Chem. Biol. 2009, 16 (2), 121-31.

(17) Ponnuswamy, N.; Bastings, M. M. C.; Nathwani, B.; Ryu, J. H.; Chou, L. Y. T.; Vinther, M.; Li, W. A.; Anastassacos, F. M.; Mooney, D. J.; Shih, W. M. Oligolysine-Based Coating Protects DNA Nanostructures from Low-Salt Denaturation and Nuclease Degradation. Nat. Commun. 2017, 8, 15654.

(18) Jungmann, R.; Steinhauer, C.; Scheible, M.; Kuzyk, A.; Tinnefeld, P.; Simmel, F. C. Single-Molecule Kinetics and SuperResolution Microscopy by Fluorescence Imaging of Transient Binding on DNA Origami. Nano Lett. 2010, 10 (11), 4756-61.

(19) Schnitzbauer, J.; Strauss, M. T.; Schlichthaerle, T.; Schueder, F.; Jungmann, R. Super-Resolution Microscopy with DNA-PAINT. Nat. Protoc. 2017, 12 (6), 1198-1228.

(20) Garcia, E.; Ismail, S. Spatiotemporal Regulation of Signaling: Focus on $\mathrm{T}$ Cell Activation and the Immunological Synapse. Int. J. Mol. Sci. 2020, 21, 9.

(21) Rothemund, P. W. Folding DNA to Create Nanoscale Shapes and Patterns. Nature 2006, 440 (7082), 297-302.

(22) Steinhauer, C.; Jungmann, R.; Sobey, T. L.; Simmel, F. C.; Tinnefeld, P. DNA Origami as a Nanoscopic Ruler for SuperResolution Microscopy. Angew. Chem., Int. Ed. 2009, 48 (47), 88703.

(23) Endesfelder, U.; Malkusch, S.; Fricke, F.; Heilemann, M. A Simple Method to Estimate the Average Localization Precision of a Single-Molecule Localization Microscopy Experiment. Histochem. Cell Biol. 2014, 141 (6), 629-38.

(24) Auer, A.; Strauss, M. T.; Strauss, S.; Jungmann, R. NanoTRON: A Picasso Module for MLP-Based Classification of Super-Resolution Data. Bioinformatics 2020, 36 (11), 3620-3622.

(25) Strauss, S.; Jungmann, R. Up to 100-Fold Speed-Up and Multiplexing in Optimized DNA-PAINT. Nat. Methods 2020, 17 (8), 789-791.

(26) Schueder, F.; Stein, J.; Stehr, F.; Auer, A.; Sperl, B.; Strauss, M. T.; Schwille, P.; Jungmann, R. An Order of Magnitude Faster DNAPAINT Imaging by Optimized Sequence Design and Buffer Conditions. Nat. Methods 2019, 16 (11), 1101-1104.

(27) Wu, N.; Czajkowsky, D. M.; Zhang, J.; Qu, J.; Ye, M.; Zeng, D.; Zhou, X.; Hu, J.; Shao, Z.; Li, B.; Fan, C. Molecular Threading and Tunable Molecular Recognition on DNA Origami Nanostructures. J. Am. Chem. Soc. 2013, 135 (33), 12172-5.

(28) Mlodzianoski, M. J.; Juette, M. F.; Beane, G. L.; Bewersdorf, J. Experimental Characterization of 3D Localization Techniques for Particle-Tracking and Super-Resolution Microscopy. Opt. Express 2009, 17 (10), 8264-77.

(29) Rieger, B.; Stallinga, S. The Lateral and Axial Localization Uncertainty in Super-Resolution Light Microscopy. ChemPhysChem 2014, 15 (4), 664-70.

(30) Bai, X. C.; Martin, T. G.; Scheres, S. H.; Dietz, H. Cryo-EM Structure of a 3D DNA-Origami Object. Proc. Natl. Acad. Sci. U. S. A. 2012, 109 (49), 20012-7.

(31) Fischer, S.; Hartl, C.; Frank, K.; Radler, J. O.; Liedl, T.; Nickel, B. Shape and Interhelical Spacing of DNA Origami Nanostructures Studied by Small-Angle X-Ray Scattering. Nano Lett. 2016, 16 (7), $4282-7$.

(32) Jungmann, R.; Avendano, M. S.; Woehrstein, J. B.; Dai, M.; Shih, W. M.; Yin, P. Multiplexed 3D Cellular Super-Resolution Imaging with DNA-PAINT and Exchange-PAINT. Nat. Methods 2014, 11 (3), 313-8.

(33) Murphy, M. C.; Rasnik, I.; Cheng, W.; Lohman, T. M.; Ha, T. J. Probing Single-Stranded DNA Conformational Flexibility Using Fluorescence Spectroscopy. Biophys. J. 2004, 86 (4), 2530-2537.

(34) Rossy, J.; Williamson, D. J.; Benzing, C.; Gaus, K. The Integration of Signaling and the Spatial Organization of the T Cell Synapse. Front. Immunol. 2012, 3, 352. 
(35) Cavalcanti-Adam, E. A.; Volberg, T.; Micoulet, A.; Kessler, H.; Geiger, B.; Spatz, J. P. Cell Spreading and Focal Adhesion Dynamics Are Regulated by Spacing of Integrin Ligands. Biophys. J. 2007, 92 (8), 2964-74.

(36) Douglas, S. M.; Marblestone, A. H.; Teerapittayanon, S.; Vazquez, A.; Church, G. M.; Shih, W. M. Rapid Prototyping of 3D DNA-Origami Shapes with CaDNAno. Nucleic Acids Res. 2009, 37 (15), 5001-6.

(37) Wagenbauer, K. F.; Engelhardt, F. A. S.; Stahl, E.; Hechtl, V. K.; Stommer, P.; Seebacher, F.; Meregalli, L.; Ketterer, P.; Gerling, T.; Dietz, H. How We Make DNA Origami. ChemBioChem 2017, 18 (19), 1873-1885. 